\title{
Cryptanalysis of the LANE Hash Function
}

\author{
Shuang Wu, Dengguo Feng, and Wenling Wu \\ State Key Lab of Information Security, Institute of Software \\ Chinese Academy of Sciences \\ Beijing 100190, China \\ \{wushuang, feng, wwl\}@is.iscas.ac.cn
}

\begin{abstract}
The LANE 4 hash function is designed by Sebastiaan Indesteege and Bart Preneel. It is now a first round candidate of NIST's SHA-3 competition. The LANE hash function contains four concrete designs with different digest length of 224, 256, 384 and 512.

The LANE hash function uses two permutations $P$ and $Q$, which consist of different number of AES1-like rounds. LANE-224/256 uses 6-round $P$ and 3-round $Q$. LANE-384/512 uses 8-round $P$ and 4-round $Q$. We will use LANE-n-(a,b) to denote a variant of LANE with a-round $P$, b-round $Q$ and a digest length $n$.

We have found a semi-free start collision attack on reduced-round LANE-256-(3,3) with complexity of $2^{62}$ compression function evaluations and $2^{69}$ memory. This technique can be applied to LANE-512- $(3,4)$ to get a semi-free start collision attack with the same complexity of $2^{62}$ and $2^{69}$ memory. We also propose a collision attack on LANE-512- $(3,4)$ with complexity of $2^{94}$ and $2^{133}$ memory.
\end{abstract}

Keywords: hash function, collision attack, rebound attack, LANE, SHA-3 candidates.

\section{Introduction}

The SHA-3 competition hosted by NIST aims to find a new cryptographic hash standard as a replacement of SHA-2. 51 of the 64 submitted designs are accepted to entered the first round. The LANE hash function is one of the first round candidates.

The attacks on widely used hash standards such as MD5[2] and SHA-1 3 . are based on differential analysis. Many of the first round candidates of SHA3 competition use AES 1 -like SPN structures and claim to resist differential attacks.

Florian Mendel et al. have proposed a new tool of "Rebound" 5 attack for cryptanalysis of AES-based designs. The main idea of rebound attack is to take advantage of weakness implied by S-box's optimal non-linearity. Random input and output differences of an S-box match with surprisingly high probability of $1 / 2$ and at least two values can be selected for each S-box. The complexity of one round in the traditional truncated differential path can be totally eliminated 
at the cost of exhausting degrees of freedom of the active state values. In this paper, we analyze reduced LANE with rebound techniques. There are other parallel works of improved rebound techniques and applications in 7/8,9].

This paper is organized as follows. In section 2, we briefly describe the LANE hash function. In section 3, we discuss inner collisions of only two lanes in the first layer P. Then semi-free start collision attacks on reduced LANE-256 are described in section 4 . Section 5 describes the attacks on reduced LANE-512. Section 6 is the conclusion.

\section{Description of LANE Hash Function}

The LANE hash function uses iterative MD structure with counters and output transformation. Digest values of LANE-224 and LANE-384 are truncated from LANE-256 and LANE-512 separately. Details of padding rules and output transformation are omitted here since they do not influence this attack.

In this section we briefly describe the compression function of LANE-256 and LANE-512. LANE-256/512 is an iterative hash function, whose compression function $f\left(H_{i-1}, M_{i}, C_{i}\right)$ processes a 512/1024-bit message block, a 512/1024bit chaining value, a 64 -bit counter, and outputs $256 / 512$-bit digest length. The chaining state $H_{i-1}$ and the message block $M_{i}$ are expanded to six 256/512bit blocks. Each block enters a different lane of $P$. Different lanes use different constants and counters. Output of the first three lanes and the last three lanes are XORed separately as input of the $Q$ permutations. At last, output of both $Q$ permutations are XORed as the next chaining value $H_{i}$. The structure of compression function in LANE is shown in Figure 1.

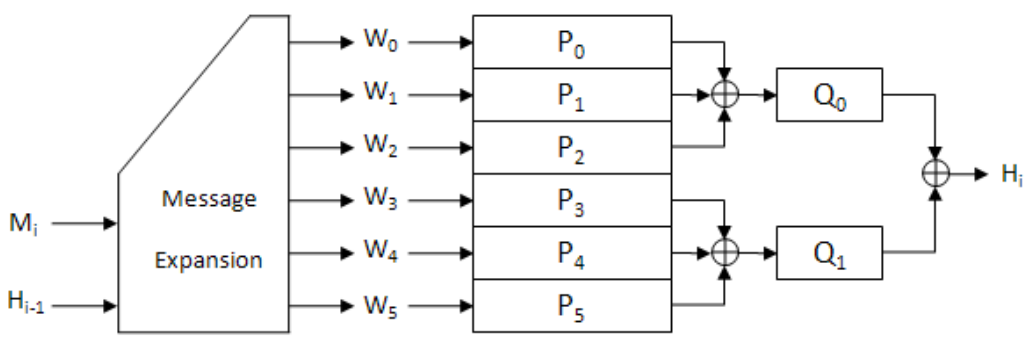

Fig. 1. The compression function of LANE

\subsection{Message Expansion}

The compression function of LANE-256 expands chaining value $H_{i-1}=h_{0} \| h_{1}$ and message block $M_{i}=m_{0}\left\|m_{1}\right\| m_{2} \| m_{3}$ to six 256-bit blocks $W_{0}, \ldots W_{5}$ as shown in equation 1 . 


$$
\begin{array}{ll}
W_{0}=h_{0} \oplus m_{0} \oplus m_{1} \oplus m_{2} \oplus m_{3} & \| h_{1} \oplus m_{0} \oplus m_{2} \\
W_{1}=h_{0} \oplus h_{1} \oplus m_{0} \oplus m_{2} \oplus m_{3} & \| h_{0} \oplus m_{1} \oplus m_{2} \\
W_{2}=h_{0} \oplus h_{1} \oplus m_{0} \oplus m_{1} \oplus m_{2} & \| h_{0} \oplus m_{0} \oplus m_{3} \\
W_{3}=h_{0} & \| h_{1} \\
W_{4}=m_{0} & \| m_{1} \\
W_{5}=m_{2} & \| m_{3}
\end{array}
$$

The message expansion in LANE-512 is analogous. The only difference is that all blocks are double-sized.

\subsection{Permutations $\mathrm{P}$ and $\mathrm{Q}$}

$P$ and $Q$ in Figure 1 are permutations with AES-like state update rounds. LANE256 uses 6-round $P$, 3-round $Q$ and LANE-512 uses 8-round $P$ and 4 -round $Q$. Each round contains five steps. One round of state update operation in LANE256 is shown in Figure 2. The difference in LANE-512 is that all operations are on four $4 \times 4$ matrices.

In this paper, we use $S_{i}$ to denote the state value after the $i$-th round. Between $S_{i}$ and $S_{i+1}$, the state values are denoted as $S_{i}^{\prime}, S_{i}^{\prime \prime}, S_{i}^{\prime \prime \prime}$ and $S_{i}^{\prime \prime \prime \prime}$ consecutively. $\triangle S_{i}$ is used to denote the XOR difference of state $S_{i}$.

The five steps of one round are:

- SB: the non-linear operation SubBytes applies an S-Box to each byte of the state. The S-box is the same as the one used in AES 1].

- SR: the cyclical permutation ShiftRows rotates the bytes of the $i$-th row leftwards by $i$ positions.

- MC: the diffusion layer MixColumns multiplies each column by a MDS matrix which is the same as the one in AES.

- AC: the constants and counter additions AddConstants and AddCounter add the round constants and the counter to the states. We use $\mathbf{A C}$ to denote both of them. The last rounds of both $P$ and $Q$ don't have AC operations. Details of the AC operations are omitted here since they have nothing to do with our attacks.

- SC: the mixing operation between different $4 \times 4$ states SwapColumns reorders the columns in the state. LANE-256 and LANE-512 use different SwapColumns operations which are shown in Figure 3.

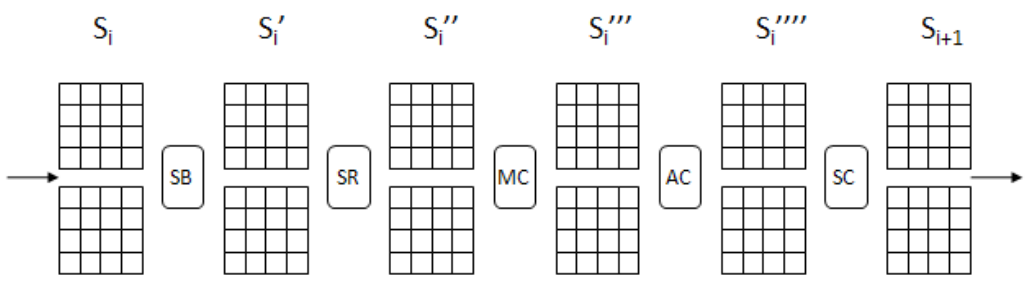

Fig. 2. One round of state update operation in LANE-256 


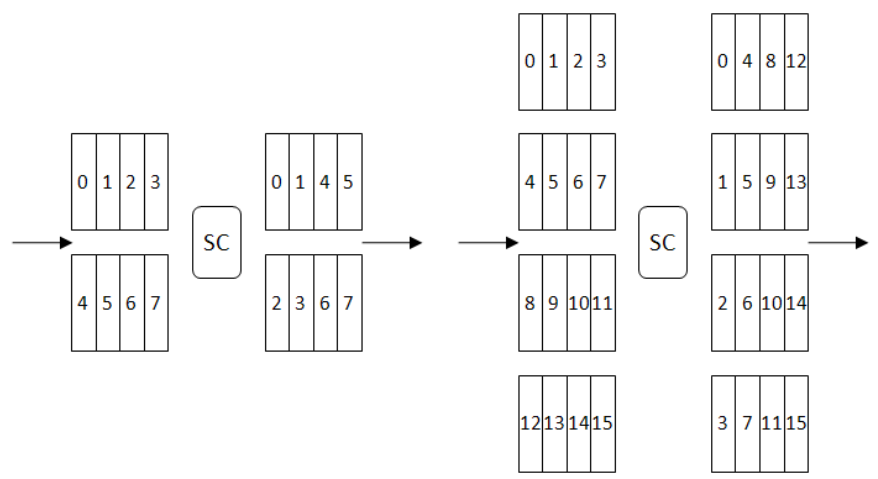

Fig. 3. SwapColumns operations used in LANE-256 and LANE-512

\section{Construct Inner Collisions Using Rebound Techniques}

The LANE hash function has six lanes in the first layer $P$. In this section, we are trying to construct collisions between only two lanes, namely the inner collisions. It's easy to see that two simultaneous inner collisions could directly lead to a full collision.

\subsection{Optimal Differential Pattern for LANE}

The message expansion used in LANE is based on a linear $(6,3,4)$-code over $G F_{4}$, which means for any possible differential path, there are at least four active lanes in the first layer $P$. Once the difference enters layer $Q$, there would be more active S-Boxes. So we want to eliminate all differences before they enter layer $Q$.

This is the best differential pattern for LANE with four active lanes $P_{1}, P_{2}, P_{4}$ and $P_{5}$. Two inner collisions in layer $P$ ensure no difference enter layer $\mathrm{Q}$ as shown in Figure 4 .

Let $\triangle m_{0}=\triangle m_{2} \neq 0$ and $\triangle h_{0}=\triangle h_{1}=\triangle m_{1}=\triangle m_{3}=0$, we have four active lanes $P_{1}, P_{2}, P_{4}$ and $P_{5}$ and the differences in $W$ are in the form of $(\triangle, 0)$ and $(0, \triangle)$. Differential paths with initial difference of $(\triangle, 0)$ and $(0, \triangle)$ behave

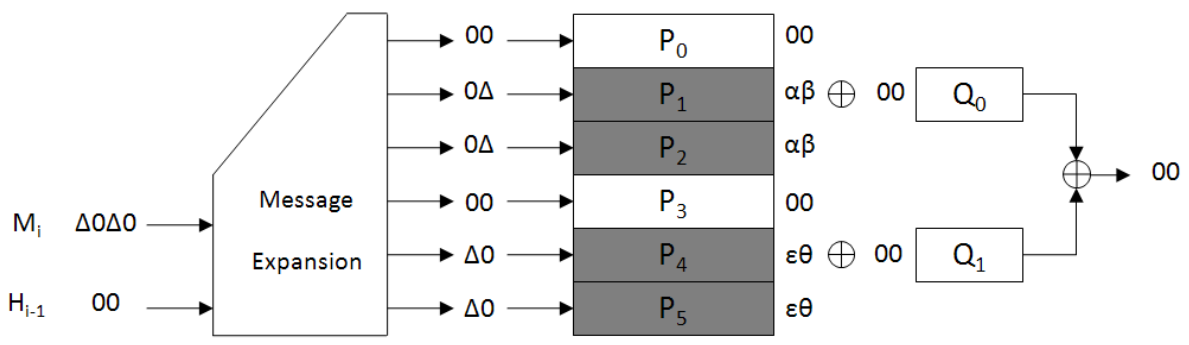

Fig. 4. Optimal differential pattern for LANE 


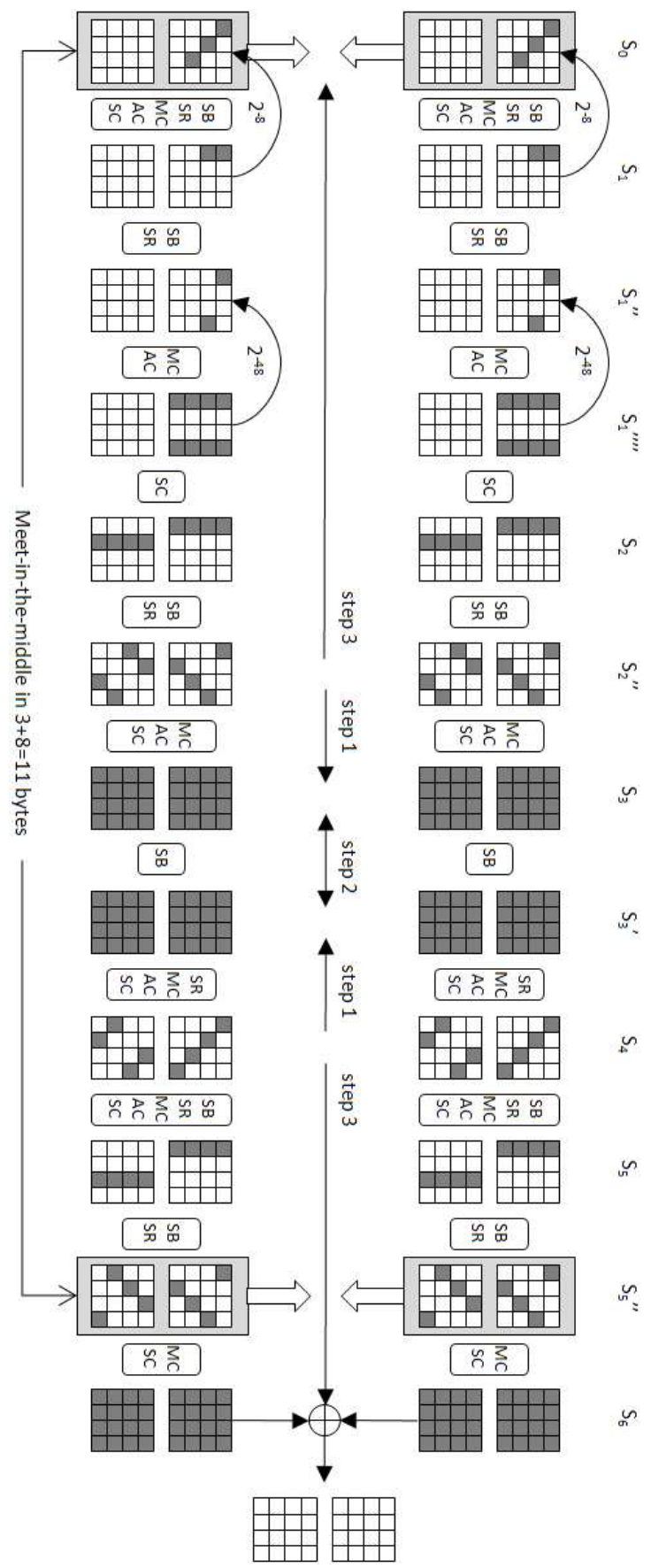

Fig. 5. Rebound differential path of an inner collision for LANE-256 
in a similar way. So we only need to consider one type of differential path in the final attack. We will talk about this in section 4.2 .

\subsection{Rebound Differential Path of Inner Collision}

In this section, we only consider an inner collision of two lanes. Using rebound techniques proposed by Florian Mendel et al. in 5, we can easily attack round 6 of layer $P$ in LANE-256 with a complexity of $2^{100}$. The differential path is shown in Figure 5 .

In traditional truncated differential path, difference propagates from initial state to hash value in forward direction. In a rebound attack, we search for an inbound differential path in internal states first. Then the outbound part can be considered as two truncated differential paths in different directions - forward and backward. Since complexity of the inbound phase can be eliminated, we only need to consider probability of the outbound phase.

Here, we briefly describe the attack of inner collision. This is similar to the attack on Grøstl[5]. For more details of rebound attack, please refer to the original paper.

Step 1: We start from choosing random differences in both $S_{2}^{\prime \prime}$ and $S_{4}$. Then compute $\triangle S_{3}$ from $\triangle S_{2}^{\prime \prime}$ and $\triangle S_{3}^{\prime}$ from $\triangle S_{4}$. These difference propagations $\triangle S_{2}^{\prime} \rightarrow \triangle S_{3}$ and $\triangle S_{3}^{\prime} \leftarrow \triangle S_{4}$ hold with probability of 1 because all operations between them are linear transformations SR, MC, AC and SC.

Step 2: We expect to find a match of possible differential character at the S-box in the third round with probability of $2^{-32}$, because random difference in input and output of an S-Box matches with probability of $1 / 2$ and there are 32 active S-boxes. Once we have found a match, we get $2^{32}$ staring points (attempts) for the outbound phase, since we have at least two values for each S-Box match. So we can generate $2^{32}$ attempts with complexity of $2^{32}$. For any $x \geq 32$ we can generate $2^{x}$ attempts with complexity of $2^{x}$.

Step 3: Each starting point (attempt) can lead to our demanded differential pattern in $S_{0}$ with a probability of $2^{-48} \times 2^{-8}=2^{-56}$. In other words, we can generate a successful attempt in one lane with complexity of $2^{56}$.

Step 4: In order to find a match in the three bytes of difference in $S_{0}$ and eight bytes in $S_{5}^{\prime \prime}$ between two lanes, we need $2^{8 \times(3+8) / 2}=2^{44}$ successful attempts in both lanes. So the complexity is $2^{56} \times 2^{44}=2^{100}$ for a inner collision with the same initial difference in both lanes. The memory requirements of step 4 is $2 \times 2^{44}=2^{45}$.

\section{Semi-free Start Collision Attack on LANE-256-(3,3)}

Even if we have successfully found two inner collisions of four lanes in layer $P$, we can not get a collision of full LANE. The problem is the message expansion since rebound attack require a full control of the state values. Four initial state values of the two inner collisions will probably lead to a contradiction since we 
have a degree of freedom for only three states, namely $\left(h_{0}, h_{1}\right),\left(m_{0}, m_{1}\right)$ and $\left(m_{2}, m_{3}\right)$.

More precisely, from two inner collisions we get the exact values of $W_{1}, W_{2}, W_{4}$ and $W_{5}$. Recall equation (1), and we can see that $W_{4}$ and $W_{5}$ can determine values of $m_{0}, m_{1}, m_{2}$ and $m_{3}$. By selecting the values of $h_{0}$ and $h_{1}$, we can change the value of $\left(h_{0} \oplus h_{1} \oplus m_{0} \oplus m_{2} \oplus m_{3}, h_{0} \oplus m_{1} \oplus m_{2}\right)$ to $W_{1}$ which we have got from the first inner collision. Since all degrees of freedom are used, we have to leave $W_{2}$ satisfied by chance.

There are 256 bits left in $W_{2}$ along with the 24-bit initial difference. We need $2^{(256+24) / 2}=2^{140}$ inner collisions in both $P_{1}, P_{2}$ and $P_{4}, P_{5}$ to find a match in $256+24=280$ bits. So in both lanes of one inner collision, we need $2^{140 / 2}=$ $2^{70}$ times more attempts. The complexity of semi-free start collision attack on LANE-256 is $2^{100} \times 2^{70}=2^{170}>2^{128}$ which exceeds the birthday bound of 256-bit hash functions and this attack fails.

\subsection{Rebound Differential Path with Partially Fixed State Values}

We are inspired by Dmitry Khovratovich et al. of their meet-in-the-middle attacks on several SHA-3 candidates [6. The idea is to fix values of certain bits to get an actually smaller size in the meet-in-the-middle part of the target state and lower the complexity.

If we fix some bytes in an AES state, they would be affected by other bytes in at most two rounds. We have got an observation that diffusion in LANE is not as efficient as in AES. Fixed values in certain positions of the initial state can proceed to the third round in both LANE-256 and LANE-512.

Combining this small observation and rebound techniques, we have found a solution for LANE-256-(3,3) as shown in Figure 6.

In this figure, one byte with a mark of " $\mathrm{X}$ " means its value can be precomputed and fixed during the attack. In our attack, we let all the $\mathrm{X}$ bytes in $S_{0}$ to be zeros and calculate values of the following ones. When we choose differences in $S_{1}^{\prime}$ and $S_{2}^{\prime \prime}$, we also set the values of fixed bytes in $S_{1}^{\prime}$ and $S_{2}^{\prime \prime}$ to what we have pre-computed.

In the four active lanes of this attack, the round constants and counters are different. So the exact values of fixed bytes in $S_{1}^{\prime}$ and $S_{2}^{\prime \prime}$ are different in four lanes. But they would all lead to zero values in the certain positions of initial states.

We also let the values of marked bytes in $h_{0}$ and $h_{1}$ to be zeros. So when we have got $W_{1}, W_{2}, W_{4}$ and $W_{5}$ from two inner collisions, we calculate the values of the non-zero bytes of $h_{0}, h_{1}, m_{0}, m_{1}, m_{2}$ and $m_{3}$ from $W_{1}, W_{4}$ and $W_{5}$. Then there are only 128 bits of state values left unsatisfied in $W_{2}$ instead of 256 bits, since all zero bytes are already satisfied in advance.

\subsection{Details of the Attack}

In this attack, We use two inner collision differential paths with initial differences of the patterns $(\triangle, 0)$ and $(0, \triangle)$ separately. If we change the position of two $4 \times 4$ matrices in the initial state of one path, the differential path don't change 


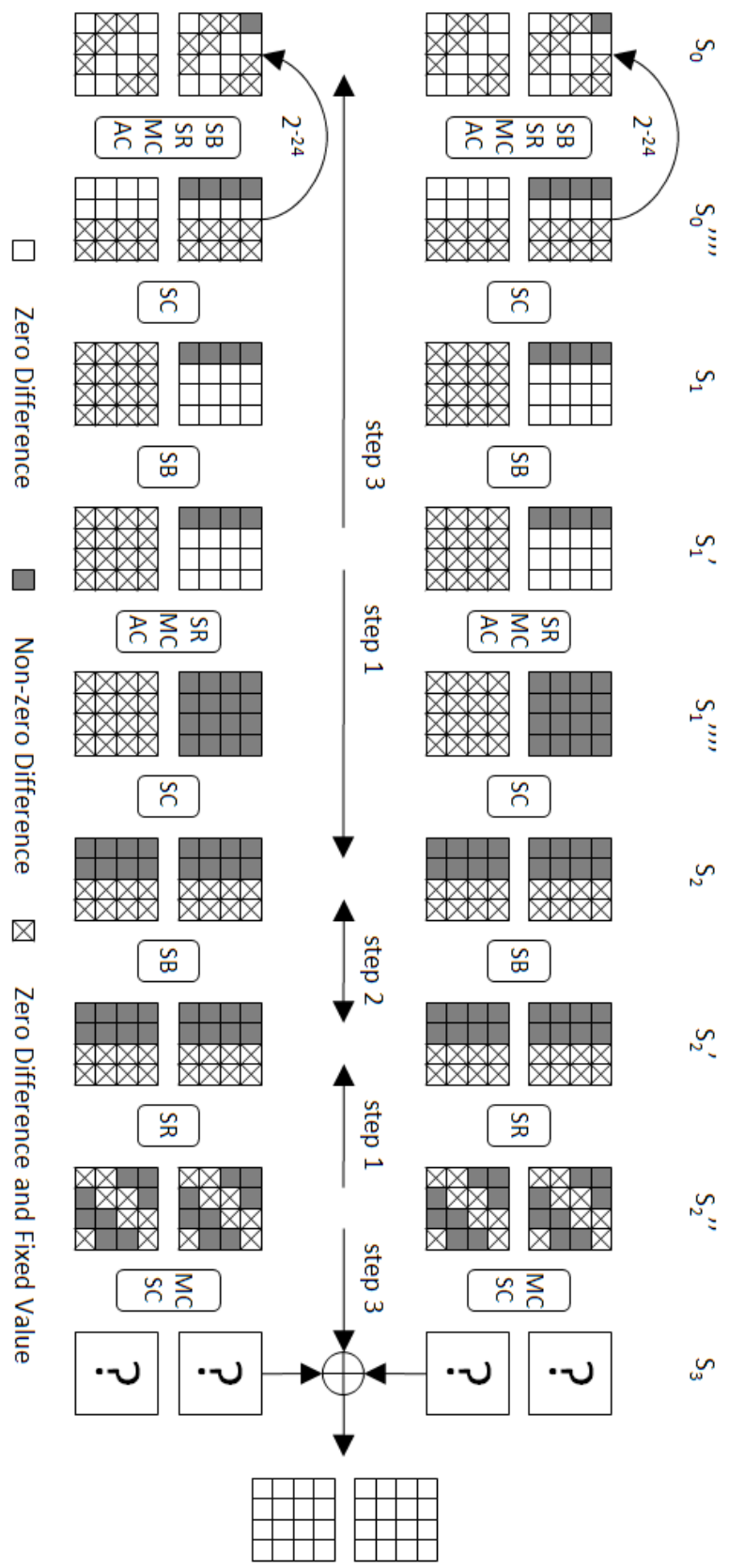

Fig. 6. Rebound differential path for LANE-256-(3,3) 


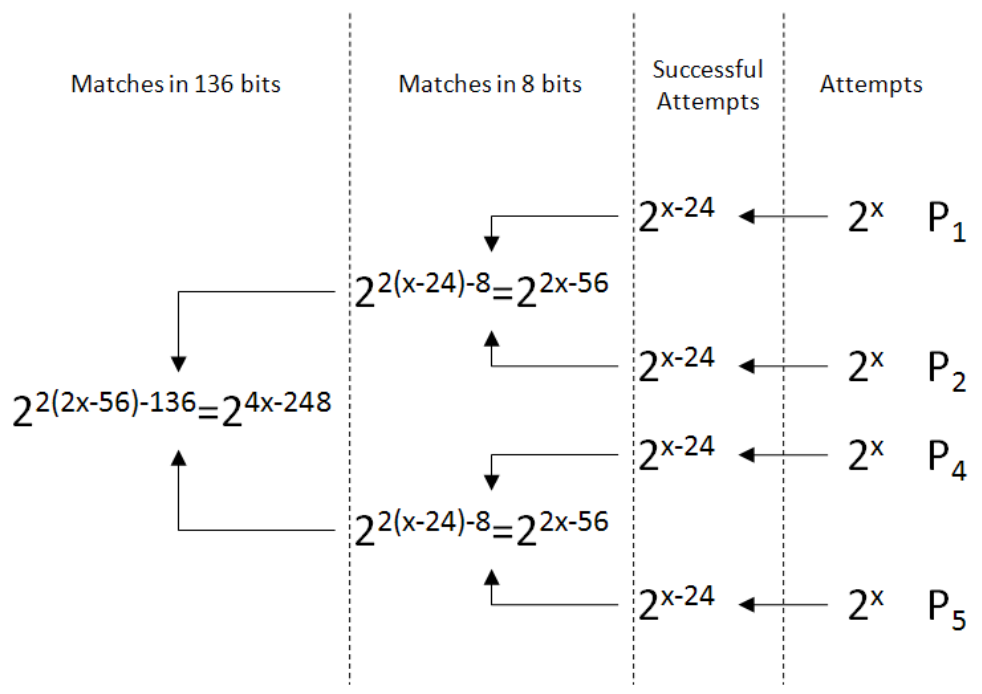

Fig. 7. Outline of the attack on LANE-256-(3,3)

substantially. Especially, the positions of fixed bytes don't change. So we consider these two differential paths equivalent and only need to analyze one of them in the following steps. Figure 7 shows outline of this attack.

This attack is described in six steps. Step 1 is the pre-computation. Steps 2 to 4 are the details in one lane of an inner collision. Step 5 is the meet-in-the-middle step of the initial difference between two lanes. Step 6 is the meet-in-the-middle step of the state values and the difference byte between two inner collisions except for the $\mathrm{X}$ bytes.

Step 1: Set all fixed bytes in the initial states in four lanes to zeros and compute the consecutive exact values of all fixed bytes in the following states.

Step 2: Choose random differences in both $S_{1}^{\prime}$ and $S_{2}^{\prime \prime}$. Here is a little difference from the attack above. We choose differences in $S_{2}^{\prime \prime}$ to be the same in both lanes of one inner collision. These two differences will remain the same when they proceed to $S_{3}$ because of the linear transformations from $S_{2}^{\prime \prime}$ to $S_{3}$. Even though we don't know the exact value of $\triangle S_{3}$ in both lanes, they must be the same and will offset each other before they enter layer $Q$.

Step 3: We expect to find a match of possible differential character at the Sbox in the second round with probability of $2^{-32}$ as in the attack above. Once we have found a match, we get $2^{32}$ staring points (attempts) for the outbound phase. Now assume that we generated $2^{x}$ attempts with complexity of $2^{x}$.

Step 4: We can find a successful attempt in one lane in every $2^{24}$ attempts. With $2^{x}$ attempts, we expect to find $2^{x-24}$ successful ones. 
Step 5: Now we have $2^{x-24}$ successful attempts in both lanes, so we can find $2^{2(x-24)-8}=2^{2 x-56}$ matches in the only one byte difference in $S_{0}$. So we have got $2^{2 x-56}$ inner collisions in $P_{1}, P_{2}$ and the same number of inner collisions in $P_{4}, P_{5}$. This step requires $4 \times 2^{x-24}$ memory.

Step 6: After we select the values of $h_{0}$ and $h_{1}$, there are 128 bits in $W_{2}$ and 8 bits in the initial difference unsatisfied. So we expect $2^{2(2 x-56)-136}=2^{4 x-248}$ matches in these 136 bits. This step requires $2 \times 2^{2 x-56}$ memory.

If $x=62$, we expect to find a final match. Memory requirements of step 5 and step 6 are $2^{40}$ and $2^{69}$. So the semi-free start collision attack on LANE-256- $(3,3)$ has an overall complexity of $2^{62}$ and requires about $2^{69}$ memory.

\section{Applications to LANE-512}

We can also use rebound techniques to find inner collisions for LANE-512. By fixing certain bytes in the state values, we can find semi-free collision and collision attacks on LANE-512-(3,4).

\subsection{Inner Collision of LANE-512}

For LANE-512, we can proceed to round 8 of $\mathrm{P}$ in an inner collision attack of two lanes. The differential path will be shown in Figure 8 as an appendix. Details of this attack is similar to inner collision attack on LANE-256 in section 3.2 .

For any given attempt, it is successful with probability of $2^{-24} \times 2^{-96}=2^{-120}$, which means we can generate one successful attempt with complexity of $2^{120}$. Then we have to match in $8 \times(16+16)=256 \mathrm{bits}$, and we need $2^{256 / 2}=2^{128}$ successful attempts in both lanes. The complexity of inner collision on LANE512 is $2^{120} \times 2^{128}=2^{248}$ and the memory requirement of meet-in-the-middle step is $2 \times 2^{128}=2^{129}$.

\subsection{Semi-free Start Collision Attack and Collision Attack on LANE-512-(3,4)}

Using the fixed bytes techniques, we can find a semi-free start collision of reduced LANE-512-(3,4) with a differential path shown in Figure 9 as an appendix. This attack is almost the same as the attack in section 4.2 with the same complexity of $2^{62}$ and $2^{69}$ memory.

As you can see in Figure 9] we can fix more bytes in the 3-round path for LANE-512. If we don't use the degrees of freedom in the initial chaining values $h_{0}$ and $h_{1}$, we have 16 more bytes in the final meet-in-the-middle part. The difference is that fixed bytes in $W_{1}$ and $W_{2}$ are not set to zeros in the marked positions. Recall equation 11, since now values of $h_{0}$ and $h_{1}$ are fixed, if we set fixed bytes of $W_{4}$ and $W_{5}$ to zeros, values of $W_{1}$ and $W_{2}$ in the marked positions are determined by the value of standard $I V=\left(h_{0}, h_{1}\right)$.

Assume that we have generated $2^{x}$ attempts in each lanes, we expect $2^{2 x-56}$ inner collisions in both $P_{1}, P_{2}$ and $P_{4}, P_{5}$. The difference is now we have to match 
$256+8=264$ bits. So we expect $2^{2 \times(2 x-56)-264}=2^{4 x-376}$ final matches with memory requirement of $2 \times 2^{2 x-56}$. If $x=94$, we expect to find one final match.

So, we have found a collision attack on LANE-512-(3,4). The complexity of collision attack on LANE-512-(3,4) is $2^{94}$ and memory requirement is $2^{133}$.

\subsection{Semi-free Start Collision Attack on LANE-512-(4,4)}

If we want to attack more than three round in $\mathrm{P}$, we can no longer use fixed values, since fixed values can only proceed to the third round. Without fixed values, we can attack LANE-512-(4,4) with a differential path shown in Figure 10 which is part of the one shown in Figure 8

Assume that we have generated $2^{x}$ attempts in each lanes, and only $2^{x-120}$ of them will be successful ones. Then we expect $2^{2(x-120)-8}=2^{2 x-248}$ inner collisions in both $P_{1}, P_{2}$ and $P_{4}, P_{5}$. Here, we have to match $512+8=520$ bits. So we expect $2^{2 \times(2 x-248)-520}=2^{4 x-1016}$ final matches with memory requirement of $2 \times 2^{2 x-248}$. If $x=254$, we expect to find one final match.

So, the complexity of semi-free start collision attack on LANE-512- $(4,4)$ is $2^{254}$ and memory requirement is $2^{261}$. Though computational complexity is less than birthday bound, memory requirement of this attack is more than $2^{256}$. This attack can be considered unsuccessful.

\section{Conclusion}

In this paper, we analyzed the LANE hash function using rebound and meet-inthe-middle techniques. We give several attacks on reduced variants of LANE-256 and LANE-512. Table 1 shows all the results of these attacks. Notation " $\dagger$ " in this table means the attack can be considered unsuccessful.

The memory requirements of all these attacks come from the meet-in-themiddle steps. But the memoryless variants seem not easy to be implemented in our attacks.

We can hardly attack more than three rounds of $\mathrm{P}$ with method of fixing certain bytes, since the fixed values can only proceed to the third round. Our attacks on reduced variants do not hurt collision resistance of full LANE.

Acknowledgments. The authors would like to thank the anonymous referees for their valuable comments. Furthermore, this work is supported by the National

Table 1. Results of collision attacks in this paper

\begin{tabular}{|c|c|c|c|c|c|}
\hline hash function & P rounds & Q rounds & collision type & complexity & memory \\
\hline \multirow{2}{*}{ LANE-256 } & 6 & - & inner collision of P & $2^{100}$ & $2^{45}$ \\
\cline { 2 - 6 } & 3 & 3 & semi-free start collision & $2^{62}$ & $2^{69}$ \\
\hline \multirow{4}{*}{ LANE-512 } & 8 & - & inner collision of P & $2^{248}$ & $2^{129}$ \\
\cline { 2 - 6 } & \multirow{2}{*}{3} & 4 & semi-free start collision & $2^{\text {62 }}$ & $2^{\text {69 }}$ \\
\cline { 2 - 6 } & 4 & 4 & semi-free start collision & $2^{254}$ & $2^{\text {253 }}$ \\
\cline { 2 - 6 }
\end{tabular}


High-Tech Research and Development 863 Plan of China (No. 2007AA01Z470), the National Natural Science Foundation of China (No. 60873259), and the National Grand Fundamental Research 973 Program of China (No. 2004CB318004).

\section{References}

1. National Institute of Standards and Technology: FIPS PUB 197, Advanced Encryption Standard (AES). Federal Information Processing Standards Publication 197, U.S. Department of Commerce (November 2001)

2. Wang, X., Yu, H.: How to break MD5 and other hash functions. In: Cramer, R. (ed.) EUROCRYPT 2005. LNCS, vol. 3494, pp. 19-35. Springer, Heidelberg (2005)

3. De Cannière, C., Rechberger, C.: Finding SHA-1 characteristics: General results and applications. In: Lai, X., Chen, K. (eds.) ASIACRYPT 2006. LNCS, vol. 4284, pp. 1-20. Springer, Heidelberg (2006)

4. Indesteege, S., Preneel, B.: The LANE hash function, http://www.cosic.esat.kuleuven.be/lane/

5. Mendel, F., Rechberger, C., Schläffer, M., Thomsen, S.S.: The Rebound Attack: Cryptanalysis of Reduced Whirlpool and Grøstl. In: Dunkelman, O. (ed.) FSE 2009. LNCS, vol. 5665, pp. 260-276. Springer, Heidelberg (2009)

6. Khovratovich, D., Nikolić, Weinmann, R.: Meet-in-the-Middle Attacks on SHA-3 Candidates. In: Dunkelman, O. (ed.) FSE 2009. LNCS, vol. 5665, pp. 228-245. Springer, Heidelberg (2009)

7. Mendel, F., Peyrin, T., Rechberger, C., Schläffer, M.: Improved Cryptanalysis of the Reduced Grøstl Compression Function, ECHO Permutation and AES Block Cipher. In: Jacobson, M.J., Rijmen, V., Safavi-Naini, R. (eds.) SAC 2009. LNCS, vol. 5867, pp. 16-35. Springer, Heidelberg (2009)

8. Lamberger, M., Mendel, F., Rechberge, C., Rijmen, V., Schläffer, M.: Rebound Distinguishers: Results on the Full Whirlpool Compression Function. In: Matsui, M. (ed.) ASIACRYPT 2009. LNCS, vol. 5912. Springer, Heidelberg (to appear, 2009)

9. Matusiewicz, K., Naya-Plasencia, M., Nikolić, I., Sasaki, Y., Schläffer, M.: Rebound Attack on the Full LANE Compression Function. In: Matsui, M. (ed.) ASIACRYPT 2009. LNCS, vol. 5912. Springer, Heidelberg (to appear, 2009) 


\section{Appendix}

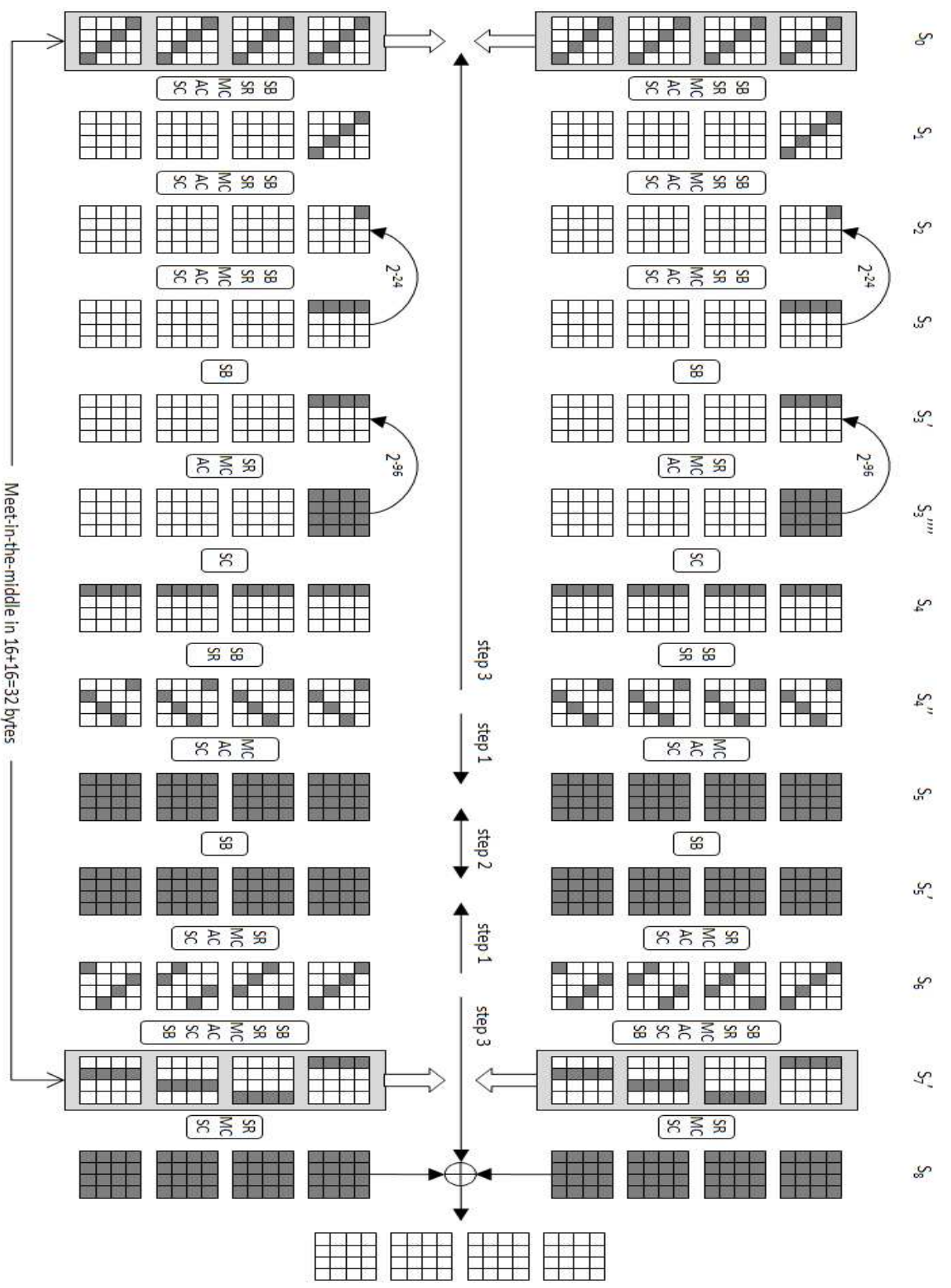

Fig. 8. Rebound differential path of inner collision for LANE-512 
w 分留

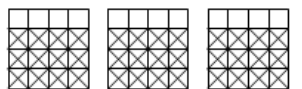
म 3
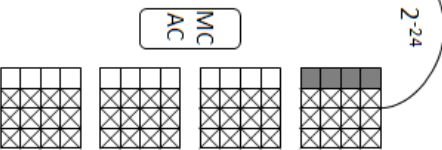

ก
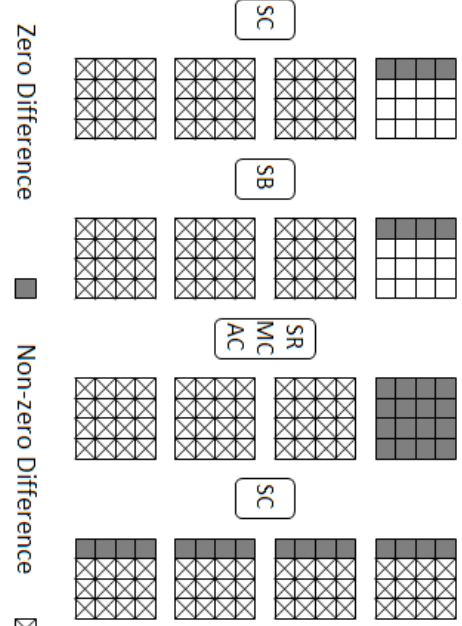

\section{$m$
$m$}
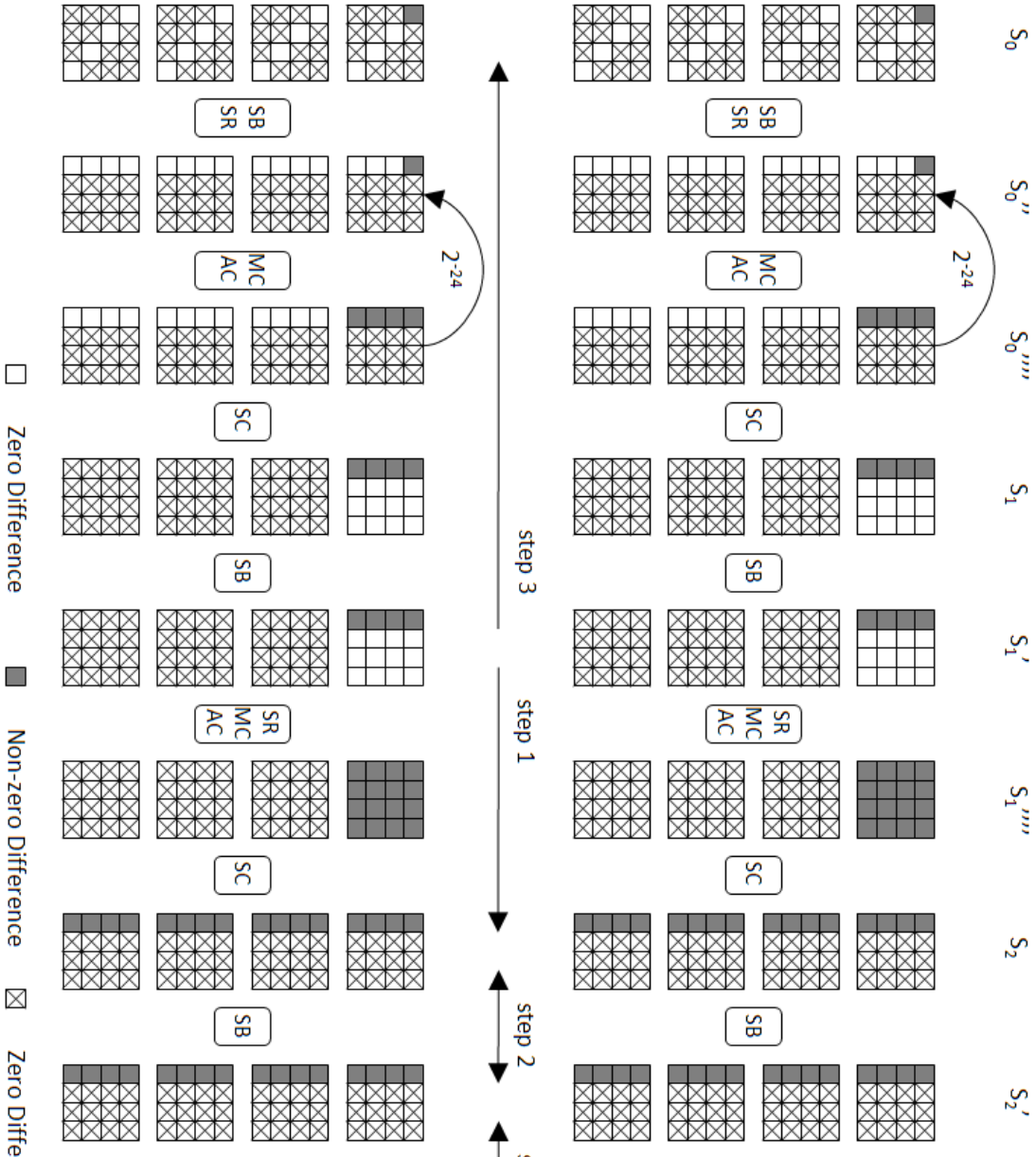

$\curvearrowleft$
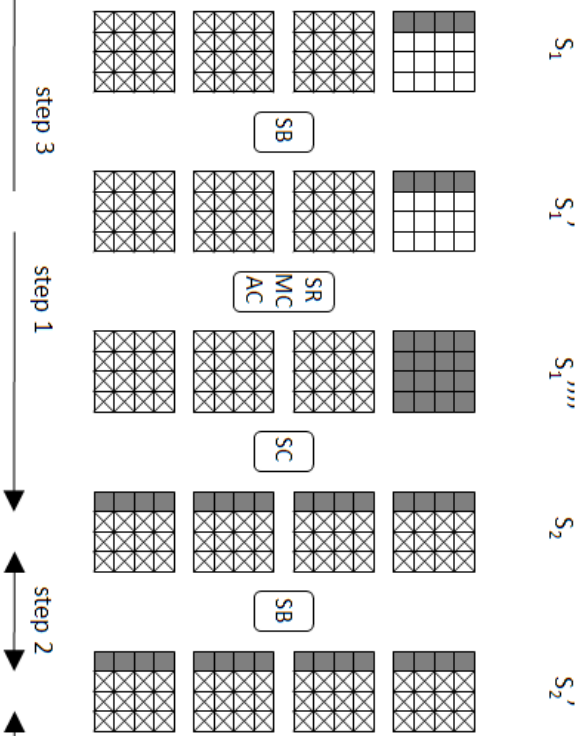

을

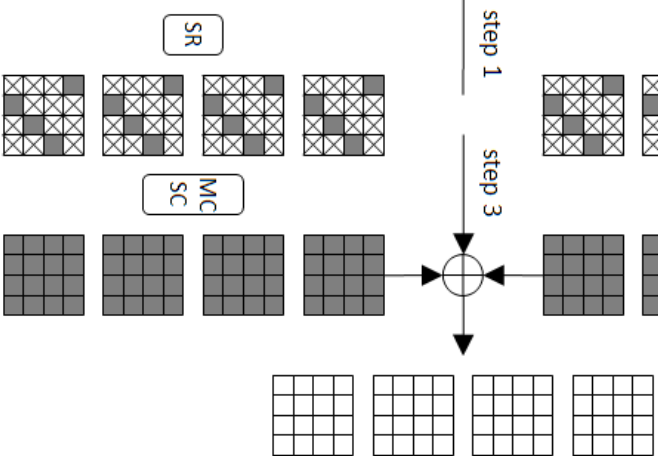

Fig. 9. Rebound differential path for LANE-512-(3,4) 


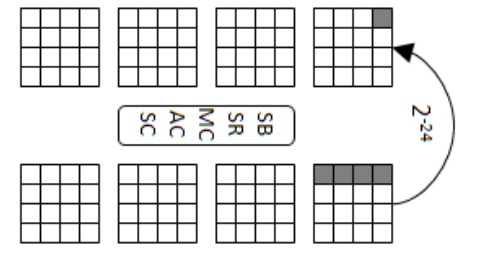

恕
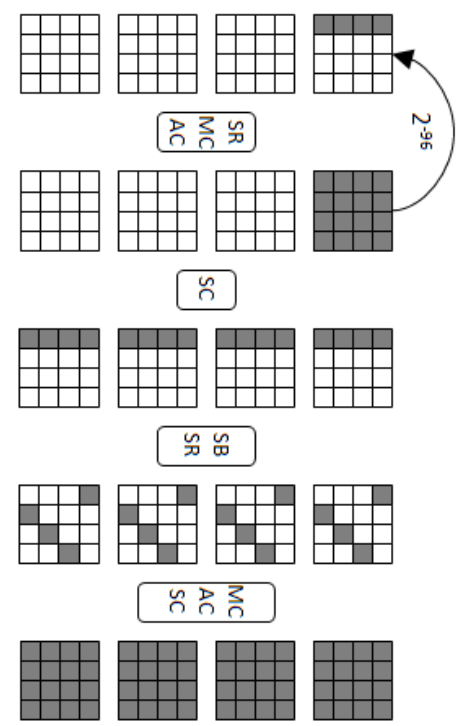

m

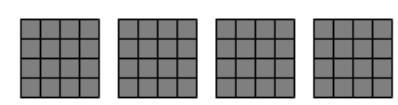

능중ㅁㄲ

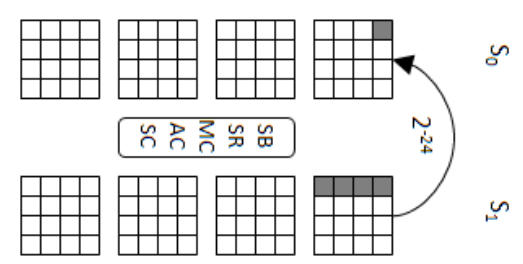

恕
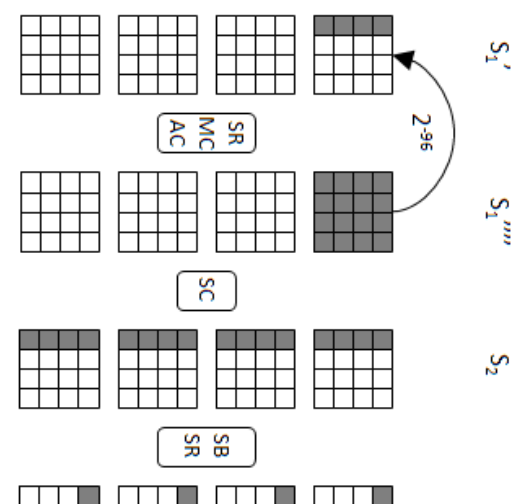

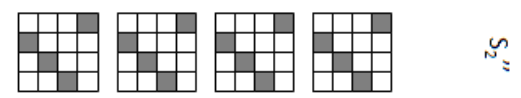
ก๊ำ

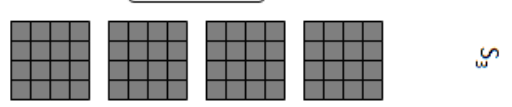

品
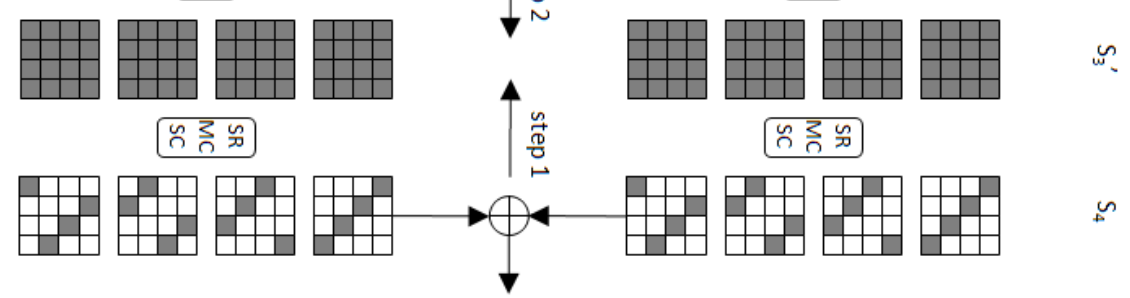

\section{四四囲四}

Fig. 10. Rebound differential path for LANE-512-(4,4) 